\title{
Un écho burlesque aux expériences de Pascal : $L a$ folie du vide (1648)
}

Jacqueline Plantié et Jean Mesnard

\section{(2) OpenEdition}

\section{Journals}

Édition électronique

URL : http://journals.openedition.org/ccibp/450

DOI : $10.4000 /$ ccibp. 450

ISSN : 2493-7460

Éditeur

Centre international Blaise Pascal

\section{Édition imprimée}

Date de publication : 4 septembre 1987

Pagination : 9-25

ISSN : 0249-6674

\section{Référence électronique}

Jacqueline Plantié et Jean Mesnard, « Un écho burlesque aux expériences de Pascal : La folie du vide

(1648) », Courrier du Centre international Blaise Pascal [En ligne], 9 | 1987, mis en ligne le 07 décembre 2015, consulté le 22 avril 2019. URL : http://journals.openedition.org/ccibp/450 ; DOI : 10.4000/ ccibp. 450

Ce document a été généré automatiquement le 22 avril 2019

Centre international Blaise Pascal 


\title{
Un écho burlesque aux expériences de Pascal : La folie du vide (1648)
}

\author{
Jacqueline Plantié et Jean Mesnard
}

1 Le petit ouvrage dont on trouvera ci-après un facsimilé intégral, publié anonymement en 1648 sous le titre La Folie du Vide ${ }^{1}$, est demeuré jusqu'ici totalement inconnu, quoiqu'il porte sur un sujet fort exploré. Il en existe au moins deux exemplaires en France, l'un à la Bibliothèque Nationale ${ }^{2}$, l'autre à la Bibliothèque Méjanes d'Aix-en-Provence ${ }^{3}$. Les deux exemplaires sont identiques. Ils comportent 44 pages, y compris, en tête, la page de titre et son verso blanc, et une dédicace de deux pages Aux Courtisans. À quoi s'ajoutent, à la fin, quatre pages non chiffrées, réservées à des pièces de vers. En tout 48 pages, de format in $-8^{\circ}$. Lors de la reliure, le premier exemplaire est demeuré isolé : il forme une mince plaquette. Le second est entré dans un recueil de quatre pièces à caractère philosophique ou scientifique, la plus récente portant la date de 1684.

2 Le texte étant immédiatement accessible, point n'est besoin de lui appliquer un long commentaire. On appréciera directement la forme très curieuse revêtue par cette critique virulente de l'hypothèse du vide, par cette défense sans nuances de la position aristotélicienne. Ni dissertation, ni discours, ni traité ; il s'agit d'un dialogue greffé sur une narration, où le récit et l'argumentation vont de pair. L'auteur professe le goût de la Cour et, quoique frais émoulu de l'Université et adepte de la physique scolastique, affirme constamment son horreur du pédantisme. De la Cour, il blâme toutefois les " cérémonies...qui, par leur sévérité, gênent l'esprit d'un homme libre et rendent les choses moins savoureuses » (p. 36). Aussi ne s'astreint-il pas à la délicatesse dans le style. S'il est capable de sérieux, il ne s'y tient guère et se complaît dans une manière de « raillerie » qui verse souvent dans la bouffonnerie et la caricature. Que de plaisanteries faciles dans la mise en parallèle de l'idée de vide dans la nature avec l'évocation d'une bourse vide (p 4), d'un ventre vide (p. 34) ou de verres vides (pp. 36-38); ou encore dans la représentation de l'adversaire d'Aristote comme " abîmé dans le vide » (p. 15). À l'époque où triomphe le burlesque, l'auteur a cru bon de suivre cette mode, sans se soucier de la finesse des effets. Des trois personnages qu'il campe, le premier, c'est-à-dire lui-même 
devenant narrateur, est gratifié d'un naturel « railleur » (p. 10), manière de s'autoriser toutes les outrances satiriques, afin d'écrire un ouvrage "sérieusement récréatif » (p. 4). Son ami Éraste, homme de Cour comme lui, en a la distinction et n'est pas trop maltraité. Toutefois, comme il est «naturaliste savant » (p. 20) et très porté à croire au vide, il est aussi dit de lui qu'il a été élevé en Allemagne, « avec plus de vin que de lait » (pp. 36-37) cette Allemagne, sans doute, qui a donné naissance à des esprits hétérodoxes tels que Paracelse. Mais la caricature se donne libre cours à propos du personnage haut en couleurs qu'est le farouche partisan du vide Théophraste, vieillard habillé à l'antique (p. 8), philosophe attaché à une antiquité suspecte, notamment à Pythagore (p. 9), pédant qui ne cesse d'entrelarder ses propos de latin, «chimiste » surtout, « le plus fameux chimiste de Paris » (p. 9), que les deux amis vont voir, au septième étage d'un « vieux logis » dans une "petite rue » (p.6), où il occupe une "salle» aux meubles de terres et de verre, fourneaux, alambics, bouteilles, flacons » (p. 7). Personnage égaré dans le monde où il vit, faisant du vide une nouvelle pierre philosophale (p. 14), se laissant aller à une ivresse qui accuse encore davantage la véritable folie dont il est atteint, la « folie du vide » Telle est le portrait-charge qui vient à la fois égayer et alourdir une critique de l'antiaristotélisme dont n'est pourtant pas exclu tout raisonnement.

3 Si le texte se suffit dans une large mesure à lui-même, il n'en soulève pas moins plusieurs énigmes, auxquelles il convient d'apporter quelques éléments de solution.

On s'interrogera d'abord sur sa date. Celle de 1648, portée sur la page de titre, n'est pas assez précise pour une époque où la question du vide évolue très rapidement, ponctuée par des échanges de correspondances scientifiques et par des publications qui se succèdent presque tous les mois. À quelle étape du déroulement de ces recherches peuton placer Le Plein du Vide? En d'autres termes, de quelles informations l'auteur de l'opuscule fait-il état?

5 Il n'est aucune raison de penser qu'il ait eu accès à des correspondances privées ou qu'il lui ait été donné de s'entretenir avec des représentants du monde savant. Les publications imprimées suffisent amplement à rendre compte des données sur lesquelles il s'appuie.

L'ouvrage auquel il se réfère le plus constamment, et celui qu'il vise - encore qu'il n'en cite ni l'auteur ni le titre - est évidemment celui de Pascal, Expériences nouvelles touchant le vide, publié en octobre $1647^{4}$. C'est là qu'il pouvait trouver la mention de l'expérience d'Italie - celle de Torricelli - qui avait donné le branle à la recherche. C'est là qu'il pouvait apprendre que cette première expérience en avait entrainé beaucoup d'autres. C'est de là qu'il pouvait tirer la description des deux expériences qu'il s'applique à présenter et à critiquer. La première est celle qui porte le $\mathrm{n}^{\circ} 3$ dans l'opuscule de Pascal ${ }^{5}$, mais c'est la plus fondamentale, puisqu'elle consiste à refaire l'expérience de Torricelli, non plus avec du « vif-argent » mais avec de l'eau ou du vin, ce qui exige l'emploi d'un très long tube. La seconde est présentée par Pascal sous le $n^{\circ} 1^{6}$. Elle se fait avec une seringue plongée dans l'eau et dont on bouche l'ouverture avec le doigt : lorsqu'on tire le piston, le doigt est attiré et le vide se fait dans le cylindre. Il n'est pas jusqu'à l'interprétation aristotélicienne des deux expériences qui n'ait pu être suggérée par l'intention que Pascal manifeste de la réfuter : qu'il s'agisse, à propos de la première expérience, de l'idée qu'un air raréfié serait entré par les pores du verre, ou que le vide apparent serait occupé par les vapeurs du liquide contenu dans le tube ; ou bien, à propos de la seconde expérience, de l'idée qu'une goutte d'eau serait entrée dans la seringue et se serait raréfiée, ou que le doigt aurait porté quelque substance qui se serait introduite dans la seringue ${ }^{7}$; qu'il s'agisse enfin de la difficulté de concilier la réalité du vide avec la transmission de la 
lumière, que celle-ci soit un accident ou une substance (p. 19) ${ }^{8}$. On notera toutefois que l'auteur s'en tient à l'argumentation la plus classique des partisans du plein : il ne souffle mot de l'hypothèse cartésienne de la matière subtile, pourtant mise en vedette dans les Expériences nouvelles.

L'ouvrage de Pascal n'est pas le seul dont La Folie du Vide suppose la connaissance. Plusieurs allusions sont faites au «docte capucin qui a fait les mêmes expériences... devant Sa Majesté polonaise, et qui les appelle démonstrations oculaires » (p. 28 ; cf. pp. 33-34), c'est-à-dire à ce Valeriano Magni qui entendait lui aussi pour fendre l'autorité d'Aristote. Il est clair que l'auteur de La Folie du Vide a lu la Demonstratio ocularis, dont il qualifie le latin de "bourru» (p.34) et dont le titre lui parait à lui seul condamner la thèse, puisqu'une démonstration doit être demandée, non aux yeux, mais à l'esprit : l'expérience ne saurait prévaloir contre la raison (p.14). Au capucin le même auteur emprunte d'ailleurs le terme de « fistule » dont il se sert parfois pour désigner le tube (pp. 12-13). Pascal ne disait rien de la Demonstratio ocularis. Mais le texte en était aisément accessible, sinon par les publications originales faites en Pologne, rares en France, du moins par la reproduction qui en fut donnée, en novembre 1647, dans un petit ouvrage destiné à l'information du grand public, l'observation touchant le vide de Marc-Antoine Dominicy 9 . Le même ouvrage fournissait le texte de la lettre de Pierre Petit à Chanut en date du 26 novembre 1646, relatant la première reprise réussie en France de l'expérience de Torricelli, par Pierre Petit et Pascal à Rouen. Voilà qui permettait de parler en connaissance de cause du « philosophe italien » (p. 10).

Tels sont des deux seuls ouvrages dont « La Folie du Vide » ait requis l'emploi. Tout le reste est issu de la culture aristotélicienne de l'auteur ou résulte de l'effort de mise en scène auquel il s'est astreint. Un doute peut toutefois s'élever quant à l'utilisation possible de l'ouvrage du fameux P. Noël, Le Plein du Vide, qui parut vers la fin de janvier $1648^{10}$. L'aristotélisme du jésuite présente assurément bien des points communs avec celui de notre auteur. Mais il offre un caractère plus moderne, notamment parce qu'il admet l'existence d'une sorte de matière subtile. De plus, dans un Avertissement, le jésuite se range à l'idée de la pesanteur de l'air. L'absence de toute allusion à cette hypothèse, de plus en plus fréquemment invoquée au cours de l'année 1648, et qui devait triompher, à l'automne de cette année, avec l'expérience du puy de Dôme, trahit l'ancienneté relative de La Folie du Vide. Reste une parenté d'expression entre le titre de cet ouvrage et celui qu'a retenu le P. Noël, en sacrifiant lui aussi à un certain goût burlesque. Même si l'on tient compte de cette parenté, on ne saurait guère aller, pour la date de publication de $L a$ Folie du Vide, au-delà des mois de février ou mars 1648.

Deuxième énigme : quel est l'auteur de notre texte? L'anonymat de la publication ne nous laisse pas totalement désarmés. Si l'Avertissement en vers placé à la fin de l'ouvrage n'est pas signé, il est suivi de trois poésies adressées à l'auteur, dont l'entourage peut être ainsi entrevu. Il est difficile d'identifier «F. du R.B. Dacq." signataire d'une première épigramme. En revanche Du Pelletier, dont un sonnet vient ensuite, est un écrivain bien connu, non seulement comme victime de Boileau, mais comme poète et comme auteur de mazarinades. Quant à François Colletet, fils de G[uillaume] Colletet, qui composa une seconde épigramme, c'était encore un tout jeune homme, puisqu'il était né en 1628, peutêtre un condisciple de l'auteur. Milieu, semble-t-il, de gens de lettres vivant, sans doute médiocrement, de leur plume et s'appliquant à saisir les occasions de se mettre en avant. Notre auteur ne songeait-il pas à s'engager dans la même carrière ? La conviction 
philosophique a sans doute moins de part, à l'origine de son ouvrage, que le désir d'attirer l'attention.

Mais il est un moyen de désigner plus précisément cet auteur. Les trois poésies qui viennent d'être citées s'adressent À Monsieur D.L.B. Ces initiales appellent un rapprochement avec la signature De La Boussière, mise à la fin d'une brochure in- $4^{\circ}$ de vingt pages, publiée en 1649 à Paris, et portant comme titre Première partie du Philosophe malotru, en vers burlesques ${ }^{11}$. Faut-il identifier les deux auteurs ? Un détail matériel nous y invite : le bandeau typographique placé au commencement du texte (p.3) est le même que celui qui figure à la même place dans $L a$ Folie du Vide (p. 5). Quant au texte de celui qui se présente lui-même comme le "philosophe malotru» - au sens étymologique de « malchanceux » - et qui emploie ses vers à tracer son portrait, il offre bien des raisons de reconnaître l'homme qui aurait écrit $L a$ Folie du Vide. Le philosophe qui se déclare «issu de noblesse » (p. 13) ne fait sans doute qu'un avec l'homme de Cour auteur de la dédicace Aux Courtisans. Par le portrait qu'il trace, il nous invite à la même identification. Il a reçu de sa famille « une bonne éducation » (p. 20); il se flatte d'une "science non commune » (p.4) ; il déclare une estime particulière aux savants, qu'il préfère même «aux plus vaillants» (p.5). Mais en dépit de sa naissance, il n'est qu'« un pauvre gentilhomme »; condamné à vivre au jour le jour, pratiquant mille petits métiers,

Tantôt en dictant mes écrits,

Tantôt transcrivant manuscrits,

Ou visitant quelque malade,

Ou bien vendant de la pommade,

Tantôt allant faire leçon

A quelque beau petit garçon,

Tantôt servant dans des villages

De médecin à petits gages,

Ou faute de meilleur emploi,

Étant mouchard aux gens du roi ;

Tantôt montrant des bagatelles

À quelques jeunes damoiselles...

(pp. 15-16).

11 Sa «noblesse » ajoute-t-il, l'a moins servi que son «adresse » (p. 20). Pourtant le métier d'écrivain qui le tente ne lui rapporte guère. S'il s'est mis à la poésie, c'est parce que la prose, dans laquelle il avait d'abord traité son sujet, ne lui laissait espérer aucune digne rétribution et que les vers lui semblaient plus estimés (p. 4). Encore n'alla-t-il pas au-delà de la première partie de son Philosophe malotru. D'où une sorte de mélancolie, le sentiment amer d'être né sous une mauvaise étoile (pp. 3, 20), dont le ton n'est pas sans affinité avec celui des premières lignes de $L a$ Folie du Vide. L'attribution de ce dernier écrit à La Boussière parait donc solidement fondée.

Mais si nous disposons d'un nom, de quelques repères sociaux, de quelques traits de caractère, il faudrait de longues recherches pour dégager les éléments d'une biographie. À en juger par le silence qui s'établit après 1649, il faut croire que la carrière, et peut-être la vie de cet auteur non dépourvu de dons, mais au goût peu sûr, auront été brèves.

Reste à se demander ce que La Folie du Vide peut apporter à la connaissance de Pascal. Elle confirme la diffusion des Expériences nouvelles en dehors du milieu purement scientifique, les controverses que l'ouvrage entraîna jusque dans la société mondaine et la tentation, éprouvée par certains écrivains mineurs, de tirer parti de ce succès en prenant position dans un sens ou dans l'autre. 
14 On a vu que l'auteur n'a disposé que d'une documentation imprimée et qu'aucune relation ne peut lui être reconnue avec vraisemblance dans le monde scientifique. En dépit de sa formation philosophique, il tient d'ailleurs à se distinguer de ce monde et fuit toute accusation de pédantisme. Ne faut-il pas aller plus loin et tenir sa création pour le fruit d'une fantaisie gratuite?

15 Comment a été construit le personnage de Théophraste ? Pascal, dans les Expériences nouvelles, est trop moderne, trop rationnel et trop soucieux de s'exprimer dans le langage de l'«honnête homme" pour avoir pu suggérer l'image de ce pédant d'un autre âge, perdu dans les fumées de son délire. Sans doute le «chimiste » présente-t-il comme siennes deux des principales expériences décrites dans le livre. Sans doute ces expériences, et les autres, exigeaient-elles l'emploi d'une multitude d'appareils qui pouvaient faire songer à l'atelier d'un alchimiste. Mais ensuite une véritable distorsion se produit. Elle n'est pas sans explication, mais ce n'est pas à Pascal de nous la fournir. Chez les alchimistes, verriers et distillateurs, qui, depuis le Moyen Age, travaillaient en marge de la scolastique officielle, se recrutaient beaucoup d'adversaires de la philosophie d'Aristote. De plus, c'étaient là, par tradition, des personnages ridicules. Voilà pourquoi le tenant du vide prend la figure de l'un d'eux. Pascal est tout à fait oublié.

L'auteur de La Folie du Vide ne va pourtant pas jusqu'à ignorer qu'il peut avoir des adversaires d'un tout autre type. C'est bien le cas de son ami Éraste, le "naturaliste » c'est-à-dire le physicien, opposé au "chimiste». Il le dépeint homme de bonne compagnie, jeune et gai, curieux de "choses nouvelles» (p.6). Qu'il en ait tracé le portrait en songeant un peu à Pascal peut se déduire d'un passage important. Lorsque Théophraste déclare « que la nature n'a pas le vide en horreur si absolument qu'elle n'en puisse souffrir aucun, mais qu'elle en souffre le moins qu'elle peut » (p. 16), c'est d'Éraste qu'il est dit avoir emprunté cette "subtilité ». Or n'est-ce pas cette thèse de l'horreur limitée du vide qui forme la conclusion, au moins provisoire, des Expériences nouvelles? Entre les deux partisans du vide, c'est en fait Éraste qui se trouve le plus expressément identifié à Pascal. Ne cherchons pas toutefois de ressemblance significative. Tout attachement à l'hypothèse du vide est dépeint comme suspect : Éraste ne le doit-il pas à son éducation allemande qui le tire du côté de Théophraste ? L'auteur de La Folie du Vide ne saurait admettre une autre rationalité que celle d'Aristote : l'esprit de la science moderne lui est inaccessible.

Que Pascal ait connu cet écrit, on ne peut guère en douter. S'il a négligé d'y répondre, c'est qu'il ne lui prêtait pas grande valeur, et qu'il en avait perçu l'intention principalement bouffonne. Lorsque, à son tour, il pratiquera le dialogue, dans Les Provinciales, il saura trouver, dans la raillerie, la justesse, la finesse et, par là-même, l'efficacité, qui avaient tant fait défaut, en 1648 , à son burlesque adversaire.

\section{NOTES}

1. Dans la présente notice, nous rétablissons l'orthographe moderne pour toutes les citations.

2. R 12577. 
3. In-8 6263.

4. On le trouvera dans Pascal, Euvres complètes, éd. Jean Mesnard, t. II, Paris, Desclée de Brouwer, 1970, pp. 493-508.

5. Ibid., pp. 502-503.

6. Ibid., p. 502.

7. Ibid., pp. 506-507.

8. Ibid., p. 508.

9. Voir Ibid., pp. 541-547

10. Sur cette date, ibid., p. 557. À titre de curiosité, sans incidence sur notre propos, on notera que, dans le recueil de la Bibliothèque Méjanes qui la contient, La folie du vide est suivi d'un Recueil de diverses expériences mécaniques et physiques tirées de plusieurs auteurs célèbres sur la possibilité du vide dans la nature : réimpression inconnue, sous la date de 1680, du Plein du vide.

11. Bibl. Nat., Ye 3173.

INDEX

Mots-clés : vide

Keywords : vacuum

\section{AUTEURS}

\section{JACQUELINE PLANTIÉ}

Historienne de la littérature française. - Agrégée de lettres. - Professeur de l'université de Provence. - Ancienne élève de l'École normale supérieure (1945)

\section{JEAN MESNARD}

Professeur émérite de littérature française du XVIIe siècle

Université de Paris-IV 